\title{
SU(4)-flavor symmetry breaking in hadron couplings
}

\author{
Gastao Krein ${ }^{* \dagger}$ \\ Instituto de Fisica Teorica, Universidade Estadual Paulista \\ Rua Dr. Bento Teobaldo Ferraz, 271 - Bloco II \\ 01140-070 São Paulo, SP - Brazil \\ E-mail: gkrein@ift.unesp.br
}

\begin{abstract}
We present results of two studies examining the accuracy of SU(4)-flavor symmetry relations between hadron couplings. Initially, we discuss results obtained with a ${ }^{3} P_{0}$ quark-pair creation model with hadron wave functions obtained from a nonrelativistic quark model. Next, we contrast the ${ }^{3} P_{0}$ results with those obtained in a framework in which all elements are constrained by studies of Dyson-Schwinger and Bethe-Salpeter equations in QCD. We focus on the three-meson couplings $\rho \pi \pi, \rho K K$, and $\rho D D$ and meson-baryon couplings $\pi N N, K \Lambda N$ and $D \Lambda_{c} N$. Within the ${ }^{3} P_{0}$ model, we find that the SU(4) breaking is at most $30 \%$ in the charm sector, for the three-meson and meson-baryon couplings. Within the relativistic Dyson-Schwinger-Bethe-Salpeter framework, the breaking is large in the charm sector, of the order of $300 \%$ for the three-meson couplings, but the predicted form factors are much softer than those usually used in meson-exchange models. Consequences of these findings for the predictions of $D N$ cross-sections and of exotic nuclear bound states of $J / \Psi$ or heavy-light $D$ and $D^{*}$ mesons are discussed.
\end{abstract}

Xth Quark Confinement and the Hadron Spectrum

$8 \ddot{o} 12$ October 2012

TUM Campus Garching, Munich, Germany

* Speaker.

${ }^{\dagger}$ A footnote may follow. 


\section{Introduction}

Effective Lagrangians based on SU(4)-flavor symmetry are widely used in studies of charmedhadron interactions with nucleons and nuclear matter. Such interactions are focus of immense contemporary interest for ongoing heavy-ion experiments and forthcoming experiments to be conducted at FAIR and JLab. The symmetry is strongly broken at the level of the QCD Lagrangian due to the large differences among quark masses of different flavors.

In the present talk, we present results of two recents studies $[1,2]$ examining the accuracy of SU(4)-flavor symmetry relations between hadron-hadron couplings, with particular interest in the couplings of charmed $D$ mesons to light mesons and nucleons. Our investigation is of interest for studies predicting low-energy $D N$ cross sections $[3,4]$ and of exotic nuclear bound states of $J / \Psi[5,6]$, or heavy-light $D$ and $D^{*}$ mesons $[7,8,9]$. Because of a complete lack of experimental information on all these reactions and nuclear states, minimally reliable calculations must be based on models constrained as much as possible by symmetry arguments, analogies with other similar processes, and the use of different degrees of freedom. Since SU(4) symmetry plays a major role in the calculations, it is of utmost importance to check its accuracy.

Specifically, we are particularly interested in examining the accuracy of the following SU(4) symmetry identities of couplings constants:

$$
\frac{g_{\pi \pi \rho}}{2}=g_{K K \rho}=g_{\bar{D} \bar{D} \rho} \quad \text { and } \quad g_{N N \pi}=g_{N \Lambda_{s} K}=g_{N \Lambda_{c} \bar{D}}
$$

Hadron-hadron couplings are determined by transition matrix elements of three-hadron states. In terms of quark-gluon degrees of freedom, the matrix elements depend on the wave functions of the hadrons involved in the transition and as such depend on the quark masses. We start with the study conducted in the context of a ${ }^{3} P_{0}$ quark-pair creation model with hadron wave functions obtained from a nonrelativistic quark model (NRQM) [1]. The results obtained with the ${ }^{3} P_{0}$ model are then contrasted with those obtained in a framework using Dyson-Schwinger-Bethe-Salpeter (DSBS) equations, in which all elements are constrained by studies in $\mathrm{QCD}$, incorporating a consistent, direct and simultaneous description of light- and heavy-quarks [2].

\section{Hadron couplings from the ${ }^{3} P_{0}$ pair-creation model}

Within the ${ }^{3} P_{0}$ quark-pair creation model, three-hadron couplings $g_{A B C}$ can be related to the matrix element of the decay process of a hadron $A$ to $B+C$ hadrons. A convenient formulation of the model is to regard the decay as due to a quark-antiquark pair creation Hamiltonian $H_{q \bar{q}}$ expressed in terms of Dirac quark fields $\psi$ as

$$
H_{q \bar{q}}=\gamma \sum_{f} 2 m_{f} \int d^{3} x\left[\bar{\psi}^{f}(\mathbf{x}) \psi^{f}(\mathbf{x})\right]_{q \bar{q}}
$$

where $\gamma$ is the pair production strength and $m_{f}$ is the mass of the produced quark and antiquark of flavor $f$ - color indices will not be indicated. The $q \bar{q}$ index in $[\bar{\psi} \psi]_{q \bar{q}}$ indicates that only the piece proportional to the product of quark and antiquark creation (or two annihilation) operators is to be taken in the product $\bar{\psi} \psi$. In order to make this explicit, let us expand the Dirac field operator in 
terms of creation and annihilation operators:

$$
\psi^{f}(\mathbf{x})=\int \frac{d^{3} p}{(2 \pi)^{3 / 2}} \sum_{s}\left[u_{s}^{f}(\mathbf{p}) q_{s}^{f}(\mathbf{p})+v_{s}^{f}(-\mathbf{p}) \bar{q}_{s}^{f \dagger}(-\mathbf{p})\right] e^{i \mathbf{p} \cdot \mathbf{x}},
$$

where, in the nonrelativistic limit, the Dirac spinors $u_{s}^{f}(\mathbf{p})$ and $v_{s}^{f}(\mathbf{p})$ are given by

$$
u_{s}^{f}(\mathbf{p})=\left(\begin{array}{c}
1-\mathbf{p}^{2} / 8 m^{f} \\
\sigma \cdot \mathbf{p} / 2 m^{f}
\end{array}\right) \chi_{s}, \quad v_{s}^{f}(\mathbf{p})=\left(\begin{array}{c}
-\sigma \cdot \mathbf{p} / 2 m^{f} \\
1-\mathbf{p}^{2} / 2 m^{f}
\end{array}\right) \chi_{s}^{c},
$$

with $\chi_{s}$ a Pauli spinor and $\chi_{s}^{c}=-i \sigma^{2} \chi_{s}^{*}$. Using these expressions in Eq. (2.1), one obtains

$$
H_{q \bar{q}}=\gamma \sum_{f} \sum_{s_{1}, s_{2}} \int d^{3} p_{1} d^{3} p_{2} \delta\left(\mathbf{p}_{1}+\mathbf{p}_{2}\right)\left[\sigma_{s_{1} s_{2}}^{c} \cdot\left(\mathbf{p}_{1}-\mathbf{p}_{2}\right)\right] q_{s_{1}}^{f \dagger}\left(\mathbf{p}_{1}\right) \bar{q}_{s_{2}}^{f \dagger}\left(\mathbf{p}_{2}\right)+\text { h.c. },
$$

where $\sigma_{s_{1} s_{2}}^{c}=\chi_{s_{1}}^{\dagger} \sigma \chi_{s_{2}}^{c}$. The matrix element of the decay $A \rightarrow B+C$ is written as

$$
\left\langle B C\left|H_{q \bar{q}}\right| A\right\rangle=\delta\left(\mathbf{P}_{A}-\mathbf{P}_{B}-\mathbf{P}_{C}\right) h_{A \rightarrow B+C},
$$

where the $\mathbf{P}_{i}, i=A, B, C$ are the momenta of the hadrons and the delta function indicates momentum conservation in the decay process. Here we will be interested in the cases where hadron $C$ is the same as hadron $A$.

One approach to define effective hadronic coupling constants was developed in the 1970s by Le Yaouanc et al. [10]: the hadron decay amplitude calculated in the quark model is equated to the corresponding emission amplitude calculated at tree level with an effective hadron Lagrangian. Specifically, let $H_{\text {eff }}$ be the Hamiltonian corresponding to the effective hadron interaction Lagrangian; then the coupling constant in the interaction Lagrangian is extracted from:

$$
\left\langle B C\left|H_{q \bar{q}}\right| A\right\rangle \equiv\left\langle B C\left|H_{e f f}\right| A\right\rangle .
$$

Of course, such an identification of matrix elements is unambiguous only when using a covariant quark model, like the one used in Ref. [2] based on a covariant DSBS framework. Within a NRQM, one simply assumes a reference frame in which the the initial hadron is at rest [10], the so-called "decay frame" [11]. For nonrelativistic kinematics and weak binding (mass of a hadron given essentially by the sum of the constituent quark masses), such frame ambiguities are expected not to be too serious at small momentum transfers [11] - we come back to this issue shortly ahead when discussing results .

In addition to the complication of an implicit frame dependence, there is at least one further problem when applying such an approach to investigate SU(4) flavor symmetry breaking in hadron couplings. While in the quark model the internal hadron structure in the decay amplitude comes in a natural way via a form factor describing the overlap of the hadrons in the decay process see Eq. (2.9), the corresponding tree level amplitude calculated with an effective Lagrangian does not come with a form factor - the form factor is introduced by hand. One consequence of this is that when calculating the tree level amplitude with an effective hadron Lagrangian, huge SU(4) symmetry breaking will be introduced from the outset via hadron masses that enter the hadron field 
operators. For example, an effective Lagrangian describing the coupling of pseudoscalar $\Phi_{P}$ to vector $\Phi_{V}^{\mu}$ fields is [3]:

$$
\mathscr{L}_{P P V}=g_{P P V} \Phi_{P}(x) \partial_{\mu} \Phi_{P}(x) \Phi_{V}^{\mu}(x) .
$$

This interaction Lagrangian gives for the matrix element of the process $P \rightarrow P+V$ the following expression

$$
h_{P \rightarrow P+V} \sim \frac{g_{P P V}}{m_{P} m_{V}^{1 / 2}},
$$

where $m_{P}$ and $m_{V}$ are the masses of the pseudocalar and vector mesons, respectively - these masses come from the meson field operators $\Phi_{P}$ and $\Phi_{V}$. Obviously, such an amplitude violates SU(4) flavor symmetry very badly, because of the huge difference in the masses of pseudoscalar mesons. For example: $m_{D} / m_{\pi} \sim 14$; since there is no corresponding compensation from a vertex form factor in the matrix element, one would have an SU(4) violation of the order of $1400 \%$ from the Lagrangian matrix element. On the other hand, in the quark model calculation of the matrix element, SU(4) symmetry breaking effects come from overall constant factors, related to parameters of the hadron wave functions, and from a form factor describing the overlap of the hadrons in the decay process - both effects come solely from the quark masses. Therefore, the naive identification of matrix elements as in Eq. (2.6) leads to nonsense when comparing different flavor sectors.

In order to avoid such problems, we do not mix descriptions; we stay within a framework that uses quark degrees of freedom only and analyze flavor symmetry breaking of hadron couplings by taking ratios of the amplitudes $h_{A \rightarrow B+C}$ for the different decays. Specifically, we define a threehadron coupling $g_{A B C}$ by the relation

$$
h_{A \rightarrow B+C}=g_{A B C} F_{A B C}\left(\mathbf{q}^{2}\right)|\mathbf{q}| Y_{11}(\hat{\mathbf{q}})
$$

where $\mathbf{q}=\mathbf{P}_{B}-\mathbf{P}_{C}$ is the relative momentum between the final hadrons (the initial hadron is at rest), $F\left(\mathbf{q}^{2}\right)$ is a form factor describing the overlap of the three hadrons in the decay process, and $Y_{11}(\hat{\mathbf{q}})$ is a spherical harmonic function - only the $Y_{11}$ appears in the couplings discussed here. There is an issue of defining the coupling at $\mathbf{q}=0$ or at the mass shell of one of the hadrons, but this will be discussed shortly ahead. To calculate the matrix element, one needs the wave functions of the hadrons calculated from the quark model Hamiltonian.

We obtain the hadron wave functions using the quark model Hamiltonian and corresponding parameters of Ref. [12]. Specifically, the Hamiltonian is given by:

$$
H=\sum_{i}\left(m_{i}+\frac{\mathbf{p}_{i}^{2}}{2 m_{i}}\right)+\sum_{i<j}\left[\frac{3 b}{4} r_{i j}-\frac{\alpha_{c}}{r_{i j}}+\frac{8 \pi \alpha_{c}}{3 m_{i} m_{j}}\left(\frac{\sigma^{3}}{\pi^{3 / 2}} e^{-\sigma^{2} r_{i j}^{2}}\right) \mathbf{S}_{i} \cdot \mathbf{S}_{j}+C\right] \sum_{a=1}^{8} F_{i}^{a} F_{j}^{a}
$$

where $r_{i j}$ is the interquark distance, $F_{i}^{a}$ is $\lambda_{i}^{a} / 2$ if $i$ is a quark and $-\lambda_{i}^{a *} / 2$ if $i$ is an antiquark, and $\lambda_{i}$ the color SU(3) Gell-Mann matrices. Since we are interested only in the hadron ground states, spin-orbit and tensor interactions are not used. The wave functions are found by diagonalizing the Hamiltonian in a finite basis of Gaussian wave functions with a common variational parameter [1]. Specifically, for the mesons, we write the spatial part of the wave function as

$$
\Phi(\mathbf{r})=\sum_{n=1}^{N} a_{n} \Phi_{n}(\mathbf{r}), \quad \text { with } \quad \Phi_{n}(\mathbf{r})=e^{-n \beta^{2} \mathbf{r}^{2} / 2},
$$


where $\mathbf{r}$ is the quark-antiquark relative coordinate and $\beta$ is the variational parameter of the quarkantiquark system. The $a_{n}$ 's are determined by solving the generalized eigenvalue problem:

$$
\sum_{n n^{\prime}}\left\langle\Phi_{n}|H| \Phi_{n^{\prime}}\right\rangle a_{n^{\prime}}=M(\beta) \sum_{n n^{\prime}}\left\langle\Phi_{n} \mid \Phi_{n^{\prime}}\right\rangle a_{n^{\prime}},
$$

where $M(\beta)$ is the mass of the meson, and $\beta$ is determined minimizing $M$. With a Gaussian basis, evaluation of the matrix elements are straightforward. For a single Gaussian, $N=1$, the r.m.s. radius of the meson $\left\langle\mathbf{r}^{2}\right\rangle^{1 / 2}$ is related to $\beta$ as $\left\langle\mathbf{r}^{2}\right\rangle^{1 / 2}=1 / \beta$. For the baryons, we use the same procedure - but the wave functions involve two relative coordinates and the evaluation of the Hamiltonian matrix element becomes more elaborate than for mesons; details will be presented elsewhere [1].

\section{Results and Perspectives}

We have calculated the meson couplings $\pi \pi \rho, K K \rho$, and $\bar{D} \bar{D} \rho$ and baryon-meson couplings $N N \pi, N \Lambda_{s} K$, abd $N \Lambda_{c} \bar{D}$. The values of the constituent quark masses used in the present work are: $m_{u}=m_{d}=0.375 \mathrm{GeV}, m_{s}=0.650 \mathrm{GeV}$, and $m_{c}=1.6 \mathrm{GeV}$. The values of the parameters of the potentials are: $\alpha_{c}=0.855, b=0.154 \mathrm{GeV}^{2}, \sigma=0.70 \mathrm{GeV}$, and $C=-0.4358 \mathrm{GeV}$. We obtain fairly stable results for the spectrum of the hadrons using a basis with 6 Gaussian functions. While the experimental masses for the mesons and light and strange baryons are well reproduced, those for the charmed baryons are reproduced within 10\%. With such a basis, the results for the different ratios of the couplings also converge well; the results are shown in Table 1.

\begin{tabular}{|c|c|c|c|c|c|c|}
\hline Basis size & $\frac{g_{\pi \pi \rho}}{2 g_{K K \rho}}$ & $\frac{g_{\pi \pi \rho}}{2 g_{\bar{D} \bar{D} \rho}}$ & $\frac{g_{K K \rho}}{g_{\bar{D} \bar{D} \rho}}$ & $\frac{g_{N N \pi}}{g_{N \Lambda_{S} K}}$ & $\frac{g_{N N \pi}}{g_{N \Lambda_{c} \bar{D}}}$ & $\frac{g_{N \Lambda_{s} K}}{g_{N \Lambda_{c} \bar{D}}}$ \\
\hline 1 & 1.04 & 0.89 & 0.86 & 1.04 & 0.89 & 1.04 \\
\hline 2 & 0.99 & 0.79 & 0.90 & 0.99 & 0.79 & 0.99 \\
\hline 3 & 1.03 & 1.03 & 1.00 & 1.03 & 1.03 & 1.03 \\
\hline 4 & 1.03 & 1.08 & 1.04 & 1.03 & 1.07 & 1.03 \\
\hline 5 & 1.04 & 1.15 & 1.11 & 1.04 & 1.15 & 1.04 \\
\hline 6 & 1.04 & 1.29 & 1.23 & 1.04 & 1.29 & 1.04 \\
\hline
\end{tabular}

Table 1: Ratios of couplings constants normalized to $F(0)=1$. In the SU(4) limit, all ratios are equal to unity.

The results shown in Table 1 indicate a small violation of the SU(4) identities when crossing from the light-flavor $u, d$ sector to the strange $s$ sector: the violation is of the order of $5 \%$ in both meson and baryon identities $g_{\pi \pi \rho} / 2 g_{K K \rho}=1$ and $g_{N N \pi} / g_{N \Lambda_{s} K}=1$. The violation is larger when moving to the charm sector, where the violation of the SU(4) identities $g_{\pi \pi \rho} / 2 g_{\bar{D} \bar{D} \rho}=1$ and $g_{N N \pi} / g_{N \Lambda_{c} \bar{D}}=1$ is of the order of $30 \%$. When crossing from the strange sector to the charm sector, while the violation in the baryon coupling is small, of the order of 5\%, in the meson sector it is somewhat larger, of the order of $20 \%$. 
One can understand the general trend of the results as follows. In the ${ }^{3} P_{0}$ decay model, the matrix element $h_{A \rightarrow A+B}$ decreases as the size (in coordinate space) of hadron $B$ decreases. For example, for the case of a single Gaussian one has the following dependence in $\beta_{P}$ and $\beta_{V}$ for the three-meson coupling $g_{P P V}-$ for $F\left(\mathbf{q}^{2}=0\right)=1$ :

$$
g_{P P V} \sim \frac{\left[1+2 m_{l} /\left(m_{h}+m_{l}\right)+2 \beta_{V} / \beta_{P}\right]}{\left(2+\beta_{V} / \beta_{P}\right)^{5 / 2}} \frac{1}{\beta_{P}^{3}}
$$

where $m_{l}$ and $m_{h}$ are the light- and heavy-flavor quark masses. For a fixed $\beta_{V}, g_{P P V}$ is a decreasing function of $\beta_{P}$; that is, as the size of the pseudoscalar meson decreases, the coupling to the vector meson decreases. The same is true for the baryon couplings.

The discussion above relies on the fact the we have defined the couplings for $F\left(\mathbf{q}^{2}=0\right)=1$. In meson exchange models, it is usual to define the couplings at the mass shell of the exchanged meson; in the present case, the $\rho$ meson. In the NRQM, one could attempt to examine the ratios of the amplitudes at $\mathbf{q}^{2}=-m_{\rho}^{2}$. The effect of this redefinition remains to be studied and will be reported elsewhere [1].

Next, we contrast the results for the three meson couplings with those of a recent study [2] conducted within in a framework in which all elements are constrained by studies of Dyson-Schwinger and Bethe-Salpeter equations in QCD and therefore incorporates a consistent and simultaneous description of light- and heavy-quarks and their associated bound states - for a recent review, see Ref. [13]. Many of the difficulties with the calculation within the NRQM, the most striking ones being the lack of covariance and the value of the constant $\gamma$ in Eq. (2.1), are not present in this approach.

As in the case of the NRQM, the focus in on the transition amplitude of an initial pseudoscalar meson to an identical meson via emission of a $\rho$ meson. The matrix element of this transition is defined as - we use a different notation from Ref. [2] to conform with the one used above:

$$
\left\langle P\left(p_{2}\right)|V(q, \lambda)| P\left(p_{1}\right)\right\rangle=g_{P P V}\left(q^{2}\right) \varepsilon_{\lambda} \cdot q,
$$

which defines the dimensionless, momentum-dependent coupling of a pseudoscalar mesons $P$ to a vector meson $V$ characterized by a polarization state $\lambda$ with four-momentum $q=p_{1}-p_{2}$. For $p_{1}^{2}=p_{2}^{2}=-m_{P}^{2}$ this is not a physical process (like in the discuss above) - note that a Euclidean metric is used here.

The calculation of $g_{P P V}\left(q^{2}\right)$ involves several interconnected steps: first, the Dyson-Schwinger equation for the propagators of quarks of different flavors are solved in way dynamical chiral symmetry in the light-quark sector is obtained (which entails the corresponding propagator must respect the chiral Ward-Takahashi indenty); next, using the derived quark propagators, Bethe-Salpeter equations are solved for the bound-state mesons and normalized such that the experimental values of masses and electroweak decays constants of the mesons are correctly reproduced; and, finally, the matrix element in Eq. (3.2) is obtained via a multidimensional integral of a triangle graph involving quark propagators and Bethe-Salpeter vertex functions. Physically motivated and controlled approximations in some of the steps above were implemented, all of them tested in several previous studies using this approach - discussion of the approximations and references to relevant work are given in Ref. [2]. In short, the results for $g_{P P V}\left(q^{2}\right)$ on the domain $q^{2}=\left\{-m_{\rho}^{2},+m_{\rho}^{2}\right\}$ can 
be fitted by the simple formula:

$$
g_{P P \rho}\left(q^{2}\right)=\frac{a_{P}-b_{P} q^{2}}{1+c_{P} q^{2}+d_{P} q^{4}},
$$

with the constants given by: $a_{\pi, K, D}=(1.84,0.94,5.05), b_{\pi, K, D}=(1.45,0.62,4.26) \mathrm{GeV}^{-2}, c_{\pi, K, D}=$ $(0.75,0.55,0.36) \mathrm{GeV}^{-2}$, and $d_{\pi, K, D}=(0.085,-0.16,-0.06) \mathrm{GeV}^{-4}$. In Fig. 1 we show momentum dependence of the ratios of coupling constants.

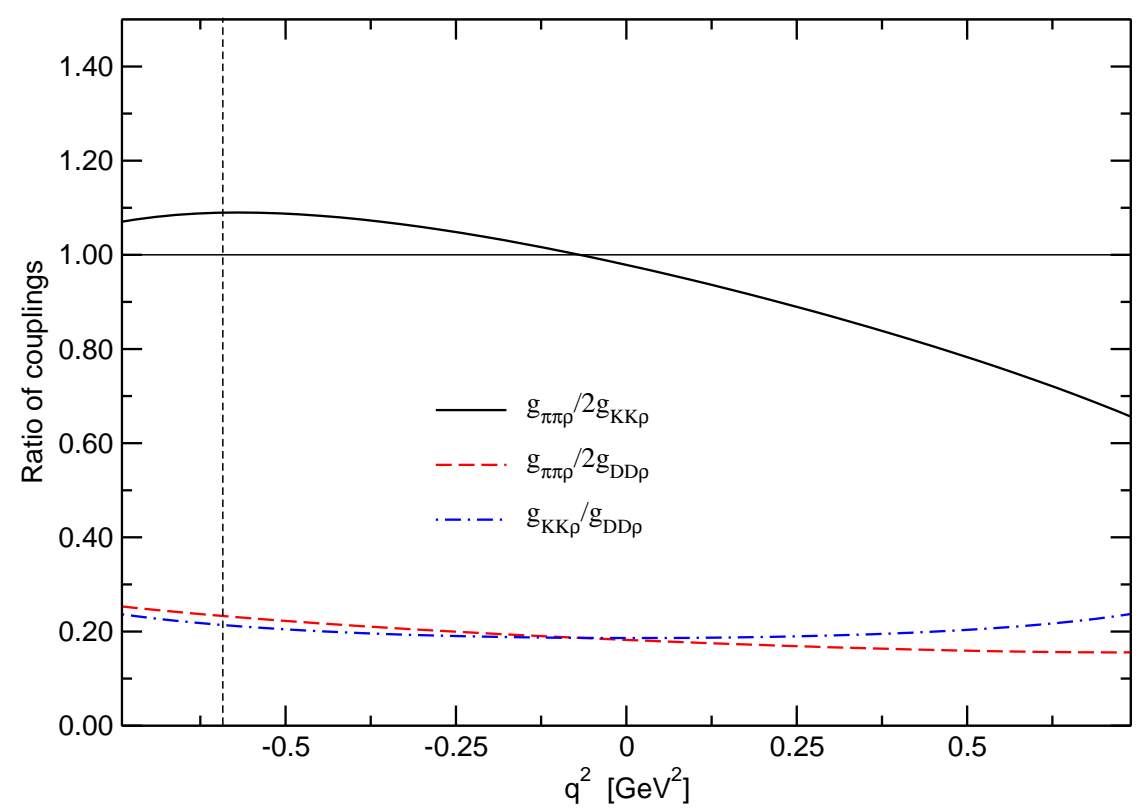

Figure 1: Momentum dependence of the ratios of coupling constants. The vertical dashed line indicates the $\rho$-meson mass shell $q^{2}=-m_{\rho}^{2}$.

As clearly seen in the figure, the ratio $g_{\pi \pi \rho} / 2 g_{K K \rho}$ is close to 1 (the $\mathrm{SU}(4)$ limit) from $q^{2}=0$ up to $\rho$-meson mass-shell. On the other hand, the other ratios are far from the SU(4) prediction, for the entire momentum range $q^{2}=\left\{-m_{\rho}^{2},+m_{\rho}^{2}\right\}$. Moreover, the violation is large, of the order of $300-400 \%$ - much larger than the violation implied by the ${ }^{3} P_{0}$ estimate (it is in the opposite direction, $g_{D D \rho}>g_{\pi \pi \rho} / 2$ and $g_{D D \rho}>g_{K K \rho}$ ). Large violations of the same order have been encountered in connection with heavy-quark symmetry in $c \rightarrow d$ transitions [14]. Large violations of SU(4) symmetry in some channels have been seen also in QCD sum rules [15] and a recent lattice calculation [16].

At first sight, such large violations of SU(4) symmetry would indicate dramatic consequences for the $D N$ interactions and also the phenomenology of exotic mesic nuclei. However, as remarked in Ref. [2], the form factors encountered here are much softer as those used in meson exchange models, e.g. in Refs. [3, 4], so that the product of a large coupling and a soft form factor lead to an inflated $\bar{D} N$ cross-section by a factor of 5 only.

Future work intends to clarify the reasons for the discrepancy of the predictions of the two approaches discussed here. 


\section{Acknowledgments}

I would like to thank Bruno El-Bennich, Lei Chang, Carlos Eduardo Fontoura, Johann Haidenbauer, Craig Roberts, and David Wilson for the collaborative work. The different parts of the work presented here were supported in part by: Conselho Nacional de Desenvolvimento Científico and Tecnológico (Brazil), Grant No. 305894/2009-9, Fundação de Amparo à Pesquisa do Estado de São Paulo (Brazil), Grant Nos. 2009/50180-0, 2009/51296-1, and 2010/05772-3; United States Department of Energy, Office of Nuclear Physics, Contract No. DEAC02-06CH11357; and Forschungszentrum Jülich GmbH.

\section{References}

[1] C.E. Fontoura, J. Haidenbauer and G. Krein, SU(4)-flavor symmetry breaking in charmed-hadron couplings, in preparation

[2] B. El-Bennich, G. Krein, L. Chang, C. D. Roberts and D. J. Wilson, Flavor SU(4) breaking between effective couplings, Phys. Rev. D 85, 031502 (2012) [arXiv:1111.3647 [nucl-th]].

[3] J. Haidenbauer, G. Krein, U. G. Meissner and A. Sibirtsev, $\bar{D} N$ interaction from meson-exchange and quark-gluon dynamics Eur. Phys. J. A 33, 107 (2007) [arXiv:0704.3668 [nucl-th] ].

[4] J. Haidenbauer, G. Krein, U. G. Meissner and A. Sibirtsev, Charmed meson rescattering in the reaction $\bar{p} d \rightarrow \bar{D} N$, Eur. Phys. J. A 37 (2008) 55 [arXiv:0803.3752 [hep-ph] ].

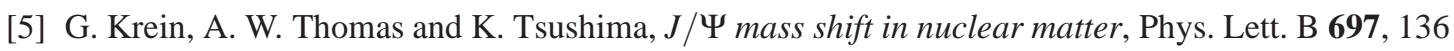
(2011) [arXiv:1007.2220 [nucl-th]].

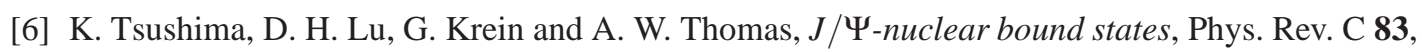
065208 (2011) [arXiv:1103.5516 [nucl-th]].

[7] K. Tsushima, D. H. Lu, A. W. Thomas, K. Saito, and R. H. Landau, Charmed mesic nuclei, Phys. Rev. C 59, 2824 (1999) [nucl-th/9810016].

[8] C. Garcia-Recio, J. Nieves and L. Tolos, D mesic nuclei, Phys. Lett. B 690, 369 (2010) [arXiv:1004.2634 [nucl-th]].

[9] C. Garcia-Recio, J. Nieves, L. L. Salcedo and L. Tolos, $D^{-}$mesic atoms, Phys. Rev. C 85, 025203 (2012) [arXiv:1111.6535 [nucl-th]].

[10] A. Le Yaouanc, L. Oliver, O. Penè and J.-C. Raynal, Naive quark pair creation model of strong interaction vertices, Phys. Rev. D 8, 2223 (1973).

[11] C. Downum, T. Barnes, J.R. Stone and E.S. Swanson, Nucleon-meson coupling constants and form-factors in the quark model, Phys. Lett. B 638, 455 (2006) [nucl-th/ 0603020 ].

[12] E. S. Swanson, Intermeson potentials from the constituent quark model, Annals Phys. 220, 73 (1992).

[13] L. Chang, C. D. Roberts and P. C. Tandy, Selected highlights from the study of mesons, Chin. J. Phys. 49, 955 (2011)[arXiv: 1107.4003 [nucl-th]].

[14] M. A. Ivanov, Yu. L. Kalinovsky and C. D. Roberts, Survey of heavy meson observables, Phys. Rev. D 60, 034018 (1999) [arXiv:nucl-th/9812063].

[15] M. E. Bracco, M. Chiapparini, F. S. Navarra and M. Nielsen, Charm couplings and form factors in QCD sum rules, Prog. Part. Nucl. Phys. 67, 1019 (2012) [arXiv:1104.2864 [hep-ph] ].

[16] K. U. Can, G. Erkol, M. Oka, A. Ozpineci and T. T. Takahashi, Vector and axial-vector couplings of D and $D^{*}$ mesons in 2+1 flavor Lattice QCD, [arXiv:1210.0869 [hep-lat] ]. 